\title{
Zweizeitige Milzruptur am 13. postraumatischen Tag nach initial unauffällig befundener CT-Untersuchung
}

\author{
Scheyerer, M J ; Schoenborn, V ; Andreisek, G ; Wanner, G A ; Werner, C M L ; Simmen, H-P
}

\begin{abstract}
Delayed splenic injuries are rare but nevertheless well known and very dangerous complications after blunt abdominal trauma. The highest incidence is reported between four and eight days after trauma; however some cases with a latent period of weeks have been published. We present a case of delayed splenic rupture 13 days after trauma where most computed tomography $(\mathrm{CT})$ examinations were interpreted as normal and present a review of the pathophysiology of delayed rupture, diagnosis and therapy.
\end{abstract}

DOI: https://doi.org/10.1007/s00113-012-2259-z

Other titles: Delayed splenic rupture 13 days post-trauma after initially inconspicuous computed tomography examination

Posted at the Zurich Open Repository and Archive, University of Zurich ZORA URL: https://doi.org/10.5167/uzh-71578

Journal Article

Published Version

Originally published at:

Scheyerer, M J; Schoenborn, V; Andreisek, G; Wanner, G A; Werner, C M L; Simmen, H-P (2013). Zweizeitige Milzruptur am 13. postraumatischen Tag nach initial unauffällig befundener CT-Untersuchung. Der Unfallchirurg, 116(6):559-562.

DOI: https://doi.org/10.1007/s00113-012-2259-z 
Unfallchirurg 2013 $\cdot 116: 559-562$

DOI 10.1007/s00113-012-2259-z

Online publiziert: 25 . Juli 2012

c) Springer-Verlag Berlin Heidelberg 2012

\section{Redaktion}

W. Mutschler, München

V. Braunstein, München
M.J. Scheyerer ${ }^{1}$ V. Schoenborn ${ }^{1}$. G. Andreisek ${ }^{2}$. G.A. Wanner ${ }^{1}$. C.M.L. Werner ${ }^{1}$. H.-P. Simmen ${ }^{1}$

${ }^{1}$ Klinik für Unfallchirurgie, Universitätsspital Zürich

${ }^{2}$ Institut für diagnostische und interventionelle Radiologie, Universitätsspital Zürich

\section{Zweizeitige Milzruptur am 13. postraumatischen Tag nach initial unauffällig befundener CT-Untersuchung}

dominaler Organe wurde zunächst nicht beschrieben (- Abb. 2). Auf der Intensivstation war der Patient allzeit, trotz der durch die Contusio cordis bedingt erhöhten Herzenzyme, kardiopulmonal stabil. Am 9. posttraumatischen Tag erfolgte die Verlegung heimatnah.

\section{Befund}

Bei Aufnahme präsentierte sich der Patient in reduziertem Allgemeinzustand, war wach und allseits orientiert. Neurologisch und kardiopulmonal war der Patient unauffällig. Der Untersuchungsbefund des Abdomens war blande.

Laborchemisch fand sich ein erhöhtes CRP $(157 \mathrm{mg} / \mathrm{l})$ und Troponin T $(0,126 \mu \mathrm{g} / \mathrm{l})$ sowie ein erniedrigtes Hämoglobin $(8,5 \mathrm{~g} / \mathrm{dl})$. Hinsichtlich des SäureBase-Haushalts sowie der Gerinnung waren die Werte unauffällig. Nach genauer retrospektiver Analyse der mitgebrachten Bildgebung stellte sich in der zweiten auswärtigen Computertomographie (CT) der Verdacht auf eine kleine Läsion der Milz $\mathrm{da}$, welche unsererseits als kontrollbedürftig eingestuft wurde (• Abb. 2).

In einer bei Ankunft erneut durchgeführten monophasische CT (AngioCT Thorax, Abdomen) stellte sich die Milz wieder als völlig unauffällig dar (• Abb. 3). Es zeigten sich lediglich neu aufgetretene Pleuraergüsse beidseits.

Stationär aufgenommen konnte der Patient wegen eines ausgeprägten Schwächegefühls nur schwer mobilisiert werden und war vorwiegend bettlägerig.
Nach zunächst unauffälligem Verlauf entwickelte der Patient am 13. posttraumatischen Tag innert kurzer Zeit das Bild eines akuten Abdomens mit Schocksymptomatik (Blutdruck 88/44 mmHg; Herzfrequenz 95/min). Das Hämoglobin fiel akut von initial 8,8 g/dl auf 2,8 g/dl.

\section{Therapie}

In der umgehend eingeleiteten explorativen Laparotomie zeigte sich eine komplette Milzruptur worauf die Splenektomie durchgeführt wurde (• Abb.4). Intraoperativ erhielt der Patient 7 Erythrozytenkonzentrate sowie 4 Konzentrate an „fresh frozen plasma“. Zur Stabilisierung wurde der Patient auf die unfallchirurgische Intensivstation verlegt. Das Abdomen wurde provisorisch durch einen abdominalen VAC-Verband verschlossen.

\section{Verlauf}

Am darauf folgenden Tage wurde bei inzwischen stabilem Patienten ein Second-look-Operation durchgeführt, welcher unauffällige intraabdominelle Verhältnisse zeigte. Das VAC-System wurde entfernt und ein sekundärer Bauchdeckenverschluss durchgeführt.

\section{Diskussion}

Die Milzverletzung zählt zu den häufigsten abdominellen Verletzungen bei stumpfem Traumata des Bauches. Die verzögerte zweizeitige Milzruptur ist da- nung mit 10 mg/Tag Arixtra (Fonaparinux) eingeleitet wurde. Eine Läsion ab- 


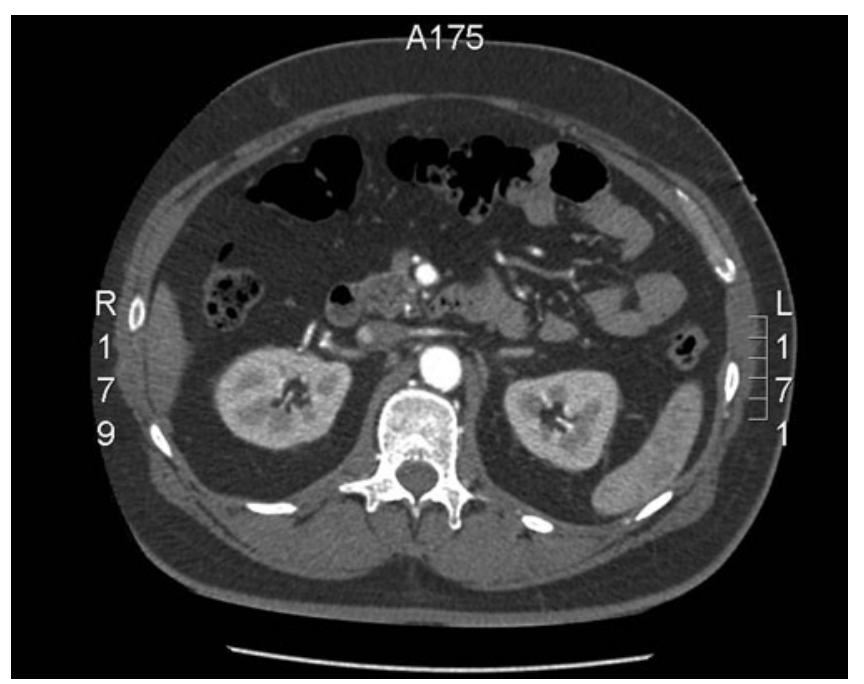

Abb. $1<$ Initiales CT zeigt eine normale Darstellung des unteren Pols der Milz

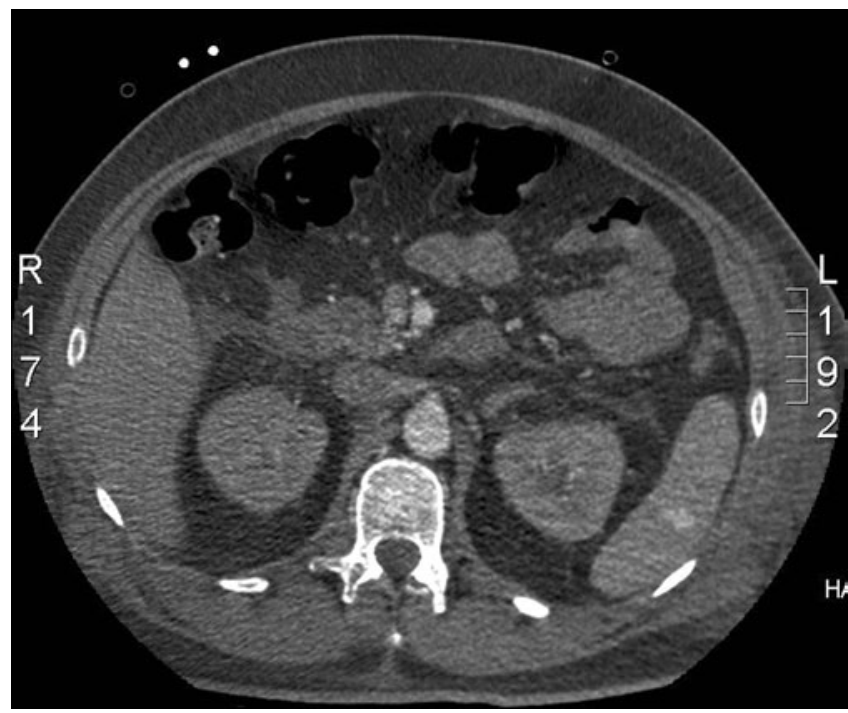

Abb. $2<$ Zweites CT zeigt eine intraparenchymale Läsion am Unterpol der Milz. Dieser Befund wurde in der initialen Beurteilung nicht beschrieben

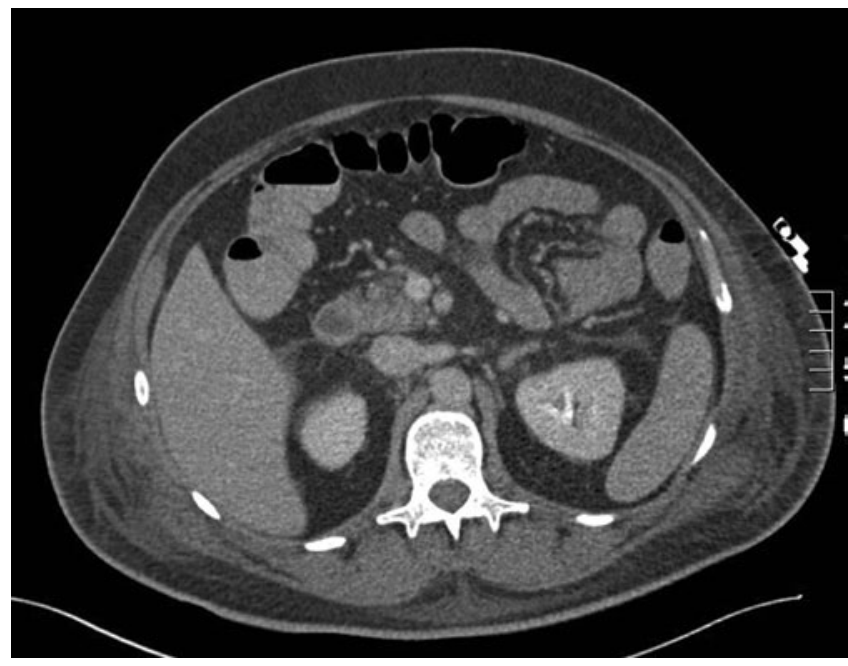

Abb. $3<$ Drittes CT zeigt eine homogene Kontrastierung der Milz. Die intraparenchymale Läsion ist nicht erkennbar

bei eine seltene, gleichwohl bekannte und potentiell sehr gefährliche Komplikation der stumpfen Milzverletzung. Ihre Inzidenz wird in der Literatur mit etwa $1-5 \%$ beschrieben [2,5]. Dabei erscheint ihr
Auftreten insbesondere bei Verletzungsmechanismen mit einer geringen Kraftwirkung, wie beispielsweise ein Sturz aus niedriger Höhe oder nach einem Kampf, besonders hoch zu sein [4]. Erstma- lig wurde die zweizeitige Milzruptur bereits im Jahre 1902 von Baudet et al. [1] beschrieben, der für sie eine Latenz von $>48 \mathrm{~h}$ nach Unfall definierte. Für gewöhnlich ereignet sie sich zwischen dem 4 . und 8. Tage posttraumatisch. Ihre Bedeutung liegt in der mit 5-15\% deutlich erhöhten Mortalität im Vergleich zu 1\% im Rahmen von akuten Ereignissen [7].

Der pathopysiologische Mechanismus der zweizeitigen Milzruptur ist noch nicht abschließend erklärt. Eine Erklärung ist, dass die einwirkende Kraft zwar das Milzparenchym schädigt und hier zu einer Lazeration führt, die Kapsel allerdings intakt bleibt. Mit fortschreitender Größe des daraus resultierenden subkapsulären Hämatoms steigt der intrasplenische Druck, worauf es zu einer Ruptur der Kapsel und zu einer aktiven Blutung kommt.

Eine weitere Hypothese ist, dass der durch das Trauma entstandene Kapselriss sowie das perisplenisches Hämatom zunächst durch die anatomische Lage zwischen Zwerchfell, Magen und linker Niere tamponiert und erst nach Tagen klinisch manifest werden [13]. Weitere diskutierte Hypothesen sind die Ruptur eines durch den Unfall entstandenen Pseudoaneurysmas eines intraparenchymatösen Gefäßes [6] oder die Ruptur von traumatisch entstandenen Pseudozysten, welche noch Jahre nach dem eigentlichen Unfall aufreißen können. Entsprechende Veränderungen konnten in der arteriellen Phase der initialen CT-Untersuchung in diesem Fall allerdings nicht dargestellt werden. Darüber hinaus existieren auch sehr fragwürdige Hypothesen wie diejenige, wo durch die vorzeitige enterale Ernährung mit fester Nahrung und die dadurch bedingte mechanische Reibung des geblähten Magens es zu einer Irritation des den Riss abdichtenden perisplenischen Hämatoms kommt. Daraus soll dann eine aktive, intraabdominale Blutung resultierten [8].

Im beschriebenen Fall konnte in der spätarteriellen Phase des initialen AngioCT ein Pseudoaneurysma ebenso wie eine aktive Blutung nicht nachgewiesen werden. In der zweiten Angio-CT-Untersuchung zeigte sich ebenfalls kein Hinweis eines Pseudoaneurysmas, allerdings Zeichen einer Parenchymläsion. Diese konn- 


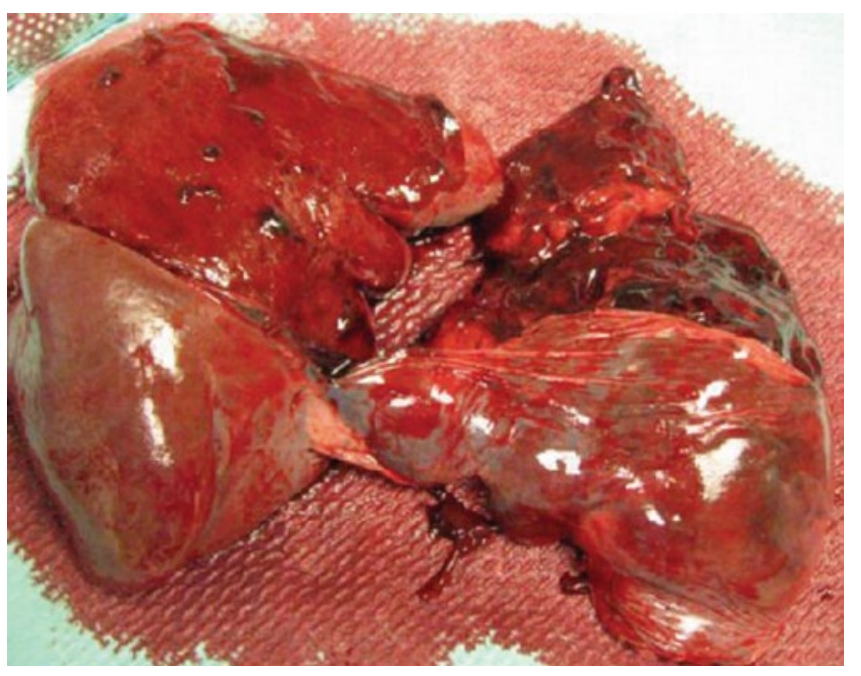

Abb. $4<$ Präparat der rupturierten Milz

te in der nach Verlegung folgenden CTUntersuchung nicht mehr dargestellt werden.

Der Einfluss der kurz nach der zweiten CT-Untersuchung begonnen antikoagulativen Therapie mit Arixtra ${ }^{\oplus}$ bleibt zu diskutieren, gleichwohl die derzeitige Literatur uns einen solchen Zusammenhang schuldig bleibt.

Hinsichtlich einer adäquaten Diagnostik ist die CT-Untersuchung seit Mitte der 1980er Jahre dennoch der Goldstandard zur Beurteilung von abdominalen Verletzungen. Inzwischen wird ihr bei der Diagnose von Milzläsionen eine Sensitivität, Spezifität sowie Treffsicherheit von 85 , 100 bzw. $97 \%$ bestätigt $[10,12]$. Neben den direkten Nachweis einer Milzverletzung, können in der CT Frakturen des linken basalen Rippenbogens, ein linksseitiger Pleuraerguss und Zwerchfellhochstand, sowie eine linksseitige Unterlappenatelektase indirekte Hinweise auf eine Milzverletzung sein. $\mathrm{Zu}$ diskutieren bleibt die Bedeutung der MRT-Untersuchung (Magnetresonanztomographie) in der Diagnostik von Milzverletzungen. Für Leberund Nierenverletzungen konnte bereits in tierexperimentellen Studien dem 3DMRT eine Überlegenheit gegenüber der CT auferlegt werden [14].

Gründe für einen falsch-negativen CTBefund, wie er bei der zweiten Untersuchung in diesem Fall auftrat, können Artefakte durch Rippen, Arme oder durch Luft im Magen sein. Bewegungsartefakte, ein falsches Timing in der Kontrastmittelgabe sowie eine falsche Protokollwahl mit fehlender arterieller Phase könnten dies ebenso beeinflussen. Auch die Latenz zwischen Unfall und initialer Bildgebung scheint eine wichtige Rolle zu spielen, wobei in der Initialphase der Untersuchungsbefund noch unauffällig sein kann [3]. Eine Theorie hierzu scheint, dass in der Frühphase ein noch nicht ausreichend eingeblutetes subkapsuläres Hämatom sich oft nicht im CT darstellen lässt [7]. Ähnliches könnte sich in dem hier vorliegenden Fall zugetragen haben. Bei unauffälligem initialen CT zeigte sich in der am 8. Tage durchgeführten Untersuchung in der gleichen spätarteriellen Phase Anzeichen einer Parenchymverletzung. Diese konnte allerdings, im 3. CT mit etwas veränderter Kontrastmittelphase (portalvenös) nicht mehr dargestellt werden.

Hinsichtlich der Therapie hat sich seit den 1970er Jahren des vergangenen Jahrhunderts ein Wechsel zum organerhaltenden Vorgehen eingestellt. Ursächlich hierfür dürfte sicherlich zum einen die Erkennung des Stellenwertes des Organs in der Immunabwehr und des durch Einzelberichten vielleicht überschätzen „Overwhelming post splenectomie infection-“ (OPSI-)Syndroms gewesen sein. Andererseits ergaben sich effiziente Möglichkeiten organerhaltend zu operieren, wie beispielsweise die Splenorraphie mittels resorbierbarem Netz, die Therapie mittels Argonbeamer, Ultraschall- oder Elektrokoagulation sowie Versiegelung mit Tachosil ${ }^{\oplus}$.

Bei Erwachsenen wird die Erfolgsquote der konservativen, milzerhaltenden Therapie mit weit über $50 \%$ beziffert [11], bei Kindern gar um einiges höher [9].
Unfallchirurg 2013 · 116:559-562

DOI 10.1007/s00113-012-2259-z

๑) Springer-Verlag Berlin Heidelberg 2012

M.J. Scheyerer · V. Schoenborn · G. Andreisek · G.A.Wanner · C.M.L. Werner · H.-P. Simmen

\section{Zweizeitige Milzruptur} am 13. postraumatischen Tag nach initial unauffällig befundener CT-Untersuchung

\section{Zusammenfassung}

Zweizeitige Milzrupturen sind seltene, gleichwohl bekannte und potentiell sehr gefährliche Komplikationen nach stumpfen abdominalen Traumata. Ihre Inzidenz ist zwischen dem 4. und 8. posttraumatischen Tag am höchsten, wobei in der Literatur auch Fälle mit einer Latenz von Wochen beschrieben werden. Wir berichten im Folgenden über eine zweizeitige Milzruptur am 13. posttraumatischen Tag, wobei die dem Ereignis vorangegangenen computertomographischen Befunde sich in der überwiegenden Zahl als unauffällig präsentierten und geben einen Überblick über die pathophysiologischen Mechanismen, die Diagnostik sowie Therapie.

\section{Schlüsselwörter}

Milzruptur, zweizeitige ·

Stumpfe abdominale Verletzung .

Computertomographie, abdominale .

Pathophysiologische Mechanismen

\section{Delayed splenic rupture 13 days post-trauma after initially inconspicuous computed tomography examination}

\section{Abstract}

Delayed splenic injuries are rare but nevertheless well known and very dangerous complications after blunt abdominal trauma. The highest incidence is reported between four and eight days after trauma; however some cases with a latent period of weeks have been published. We present a case of delayed splenic rupture 13 days after trauma where most computed tomography (CT) examinations were interpreted as normal and present a review of the pathophysiology of delayed rupture, diagnosis and therapy.

\section{Keywords}

Splenic rupture, delayed .

Blunt abdominal trauma .

Computed tomography, abdominal .

Pathophysiological mechanisms 


\section{Fazit für die Praxis}

Die zweizeitige Milzruptur am 13. posttraumatischen Tag ist ein sehr seltenes, wenn auch - wie dieser Fall zeigt - mögliches Ereignis. Selbst wenn es wie hier initial keine Anzeichen einer Verletzung des Organs gab, sehen wir bei Patienten mit Verdacht oder - aufgrund des Unfallmechanismus - der Möglichkeit einer Verletzung der Milzkontrolluntersuchungen als unerlässlich an.

Darüber hinaus lehrt uns dieser Fall allerdings auch, dass selbst die CT, welche derzeit der Goldstandard in der Diagnostik ist, nicht mit letzter Sicherheit eine Läsion darstellt und das künftig bei unklaren Fällen womöglich ein Umdenken hin zur Kernspintomographie stattfinden muss. Ihr Vorteil hinsichtlich Sensitivität und Spezifität muss in Studien allerdings noch belegt werden. Bei Patienten mit dem CT-Verdacht auf eine Läsion der Milz denken wir, dass eine Hospitalisation von mindestens
8 Tagen bei unauffälligem Verlauf notwendig zu sein scheint. Eine gründliche Aufklärung des Patienten darüber hinaus ist selbstredend unerlässlich.

\section{Korrespondenzadresse}

\section{Dr. M.J. Scheyerer}

Klinik für Unfallchirurgie,

Universitätsspital Zürich

Rämistraße 100, CH-8091 Zürich

Schweiz

maxjoseph.scheyerer@usz.ch

Interessenkonflikt. Der korrespondierende Autor gibt für sich und seine Koautoren an, dass kein Interessenkonflikt besteht.

\section{Literatur}

1. Baudet $R$ (1907) Ruptures de la rate. Med Pract 3:565

2. Cocanour CS, Moore FA, Ware DN et al (1998) Delayed complications of nonoperative management of blunt adult splenic trauma. Arch Surg 133:619-624

3. Fagelman D, Hertz MA, Ross AS (1985) Delayed development of splenic subcapsular hematoma: CT evaluation. J Comput Assist Tomogr 9:815-816

4. Farhat GA, Abdu RA, Vanek VW (1992) Delayed splenic rupture: real or imaginary? Am Surg 58:340-345

5. Hassan R, Abd Aziz A, Md Ralib AR et al (2011) Computed tomography of blunt spleen injury: a pictorial review. Malays J Med Sci 18:60-67
6. Hiraide A, Yamamoto H, Yahata Ket al (1994) Delayed rupture of the spleen caused by an intrasplenic pseudoaneurysm following blunt trauma: case report. J Trauma 36:743-744

7. Kluger Y, Paul DB, Raves JJ et al (1994) Delayed rupture of the spleen - myths, facts, and their importance: case reports and literature review. J Trauma 36:568-571

8. Kodikara S, Sivasubramanium M (2009) Mechanisms of delayed splenic rupture: a new hypothesis. Leg Med (Tokyo) 11(Suppl 1):515-517

9. Kristoffersen KW, Mooney DP (2007) Long-term outcome of nonoperative pediatric splenic injury management. J Pediatr Surg 42:1038-1041

10. Marmery $\mathrm{H}$, Shanmuganathan $\mathrm{K}$, Mirvis SE et al (2008) Correlation of multidetector CT findings with splenic arteriography and surgery: prospective study in 392 patients. J Am Coll Surg 206:685-693

11. Myers JG, Dent DL, Stewart RM et al (2000) Blunt splenic injuries: dedicated trauma surgeons can achieve a high rate of nonoperative success in patients of all ages. J Trauma 48:801-806

12. Salimi J, Bakhtavar K, Solimani M et al (2009) Diagnostic accuracy of CT scan in abdominal blunt trauma. Chin J Traumatol 12:67-70

13. Simpson RA, Ajuwon R (2001) Occult splenic injury: delayed presentation manifesting as jaundice. Emerg Med J 18:504-505

14. Weishaupt D, Hetzer FH, Ruehm SG et al (2000) Three-dimensional contrast-enhanced MRI using an intravascular contrast agent for detection of traumatic intra-abdominal hemorrhage and abdominal parenchymal injuries: an experimental study. Eur Radiol 10:1958-1964

\section{回 \\ In 66 Zeitschriften online recherchieren: Ihre e.Bibliothek Orthopädie/Unfallchirurgie}

Das e.Med-Komplettpaket von Springer Medizin bietet Ihnen Zugang zu 66 deutschsprachigen und internationalen Zeitschriften im Bereich Orthopädie/Unfallchirurgie. Über die komfortable Suchfunktion gelangen Sie direkt zu den gewünschten Beiträgen und können diese anschließend im Volltext nutzen. Mit der Merkfunktion können Sie sich ein persönliches Beitragsarchiv zusammenstellen und Beiträge auf Wunsch kommentieren. Insgesamt umfasst e.Med Beiträge aus 400 Zeitschriften aus allen medizinischen Fachgebieten.

\section{Zeitschriftenauswahl Orthopädie/Unfallchirurgie}

Internationale Titel

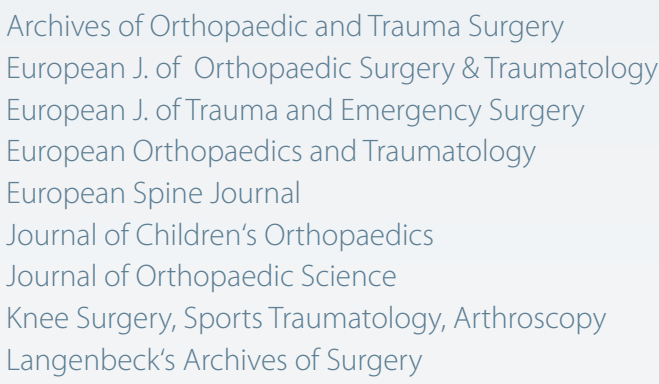

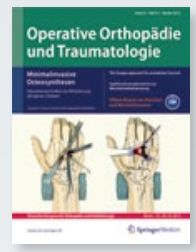
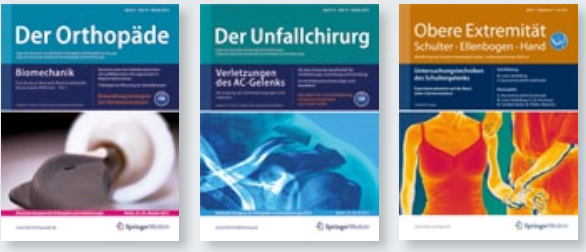

Deutschsprachige Titel

Arthroskopie

Der Orthopäde

Der Unfallchirurg

Manuelle Medizin

Obere Extremität

Operative Orthopädie und Traumatologie

Orthopädie \& Rheuma

Trauma und Berufskrankheit

Zeitschrift für Rheumatologie 